\title{
A clinical decision rule to predict intracranial hypertension in severe traumatic brain injury
}

\author{
Aziz S. Alali, MD, PhD, ${ }^{1}$ Nancy Temkin, PhD, ${ }^{1,2}$ Jason Barber, MS, ${ }^{1}$ Jim Pridgeon, MHA, ${ }^{1}$ \\ Kelley Chaddock, BA, ${ }^{1}$ Sureyya Dikmen, PhD, ${ }^{1,3}$ Peter Hendrickson, PhD, ${ }^{1}$ Walter Videtta, MD, ${ }^{4}$ \\ Silvia Lujan, MD, ${ }^{5}$ Gustavo Petroni, MD, ${ }^{5}$ Nahuel Guadagnoli, MD, ${ }^{6}$ Zulma Urbina, MD, ${ }^{7}$ and \\ Randall M. Chesnut, MD ${ }^{1,8}$
}

\begin{abstract}
1'Department of Neurological Surgery, University of Washington, Harborview Medical Center; Departments of ${ }^{2}$ Biostatistics, ${ }^{3}$ Rehabilitation Medicine, and ${ }^{8}$ Orthopaedics and Sports Medicine, University of Washington, Seattle, Washington; ${ }^{4}$ Hospital Nacional Profesor Alejandro Posadas, Buenos Aire; ${ }^{5} \mathrm{Hospital}$ de Emergencias Dr. Clemente Alvarez, Rosario; ${ }^{6} \mathrm{Hospital}$ Emergencia, Hospital Privado de Rosario, Rosario, Argentina; and ${ }^{7}$ Hospital Erasmo Meoz, Cucuta, Colombia
\end{abstract}

OBJECTIVE While existing guidelines support the treatment of intracranial hypertension in severe traumatic brain injury (TBI), it is unclear when to suspect and initiate treatment for high intracranial pressure (ICP). The objective of this study was to derive a clinical decision rule that accurately predicts intracranial hypertension.

METHODS Using Delphi methods, the authors identified a set of potential predictors of intracranial hypertension and a clinical decision rule a priori by consensus among a group of 43 neurosurgeons and intensivists who have extensive experience managing severe TBI without ICP monitoring. To validate these predictors, the authors used data from a Latin American trial ( $n=150$; BEST TRIP). To report on the performance of the rule, they calculated sensitivity, specificity, and positive and negative predictive values with $95 \%$ confidence intervals. In a secondary analysis, the rule was validated using data from a North American trial ( $n=131$; COBRIT).

RESULTS The final predictors and the clinical decision rule were approved by $97 \%$ of participants in the consensus working group. The predictors are divided into major and minor criteria. High ICP would be considered suspected in the presence of 1 major or $\geq 2$ minor criteria. Major criteria are: compressed cisterns (CT classification of Marshall diffuse injury [DI] III), midline shift > $5 \mathrm{~mm}$ (Marshall DI IV), or nonevacuated mass lesion. Minor criteria are: Glasgow Coma Scale (GCS) motor score $\leq 4$, pupillary asymmetry, abnormal pupillary reactivity, or Marshall DI II. The area under the curve for the logistic regression model that contains all the predictors was 0.86 . When high ICP was defined as $>22$ $\mathrm{mm} \mathrm{Hg}$, the decision rule performed with a sensitivity of $93.9 \%(95 \% \mathrm{Cl} 85.0 \%-98.3 \%)$, a specificity of $42.3 \%(95 \% \mathrm{Cl}$ $31.7 \%-53.6 \%$ ), a positive predictive value of $55.5 \%$ (95\% $\mathrm{Cl} 50.7 \%-60.2 \%)$, and a negative predictive value of $90 \%$ (95\% Cl 77.1\%-96.0\%). The sensitivity of the clinical decision rule improved with higher ICP cutoffs up to a sensitivity of $100 \%$ when intracranial hypertension was defined as ICP > $30 \mathrm{~mm} \mathrm{Hg}$. Similar results were found in the North American cohort.

CONCLUSIONS A simple clinical decision rule based on a combination of clinical and imaging findings was found to be highly sensitive in distinguishing patients with severe TBI who would suffer intracranial hypertension. It could be used to identify patients who require ICP monitoring in high-resource settings or start ICP-lowering treatment in environments where resource limitations preclude invasive monitoring.

Clinical trial registration no.: NCT02059941 (clinicaltrials.gov).

https://thejns.org/doi/abs/10.3171/2018.4.JNS173166

KEYWORDS traumatic brain injury; intracranial pressure; clinical decision rule; clinical prediction rule; trauma

ABBREVIATIONS AUC = area under the curve; BEST TRIP = Benchmark Evidence from South American Trials: Treatment of Intracranial Pressure; $\mathrm{Cl}=$ confidence interval; $\mathrm{COBRIT}=$ Citicoline Brain Injury Treatment Trial; DI = diffuse injury; GCS = Glasgow Coma Scale; ICP = intracranial pressure; TBI = traumatic brain injury. SUBMITTED December 17, 2017. ACCEPTED April 5, 2018.

INCLUDE WHEN CITING Published online September 28, 2018; DOI: 10.3171/2018.4.JNS173166. 
$\mathrm{T}$ RAUMATIC brain injury (TBI) is a pressing public health and medical problem worldwide. It constitutes the leading cause of injury-related deaths and loss of human potential. ${ }^{18}$ Recent estimates suggest that more than 50 million people worldwide are affected by TBI every year. ${ }^{8}$ The burden of TBI is substantially higher in low- and middle-income countries, which have more prevalent risk factors for TBI and inadequately resourced health systems to address its profound consequences. ${ }^{11}$

Severe TBI accounts for about $10 \%$ of all TBIs but it contributes the greatest proportion of death, disability, and TBI-related costs. ${ }^{9,21}$ Among those who sustain severe TBI, the majority of deaths are associated with raised intracranial pressure (ICP)..$^{17,19}$ In addition, patients who respond to ICP-lowering treatment have better outcomes than those with refractory intracranial hypertension. ${ }^{7}$ Therefore, a fundamental focus of acute TBI care continues to be the alleviation of intracranial hypertension.

The most recent update of the Brain Trauma Foundation guidelines offers an array of ICP-lowering treatment options. ${ }^{3}$ However, it remains unclear when to suspect and initiate treatment for high ICP. The recent update did not carry forward the indications for initiation of ICP monitoring that were suggested by older guidelines. ${ }^{1,3}$ Those indications were derived from descriptive studies of patient characteristics associated with risk of raised ICP and were not validated. ${ }^{1,3,15}$ Although continuous invasive ICP monitoring may facilitate the diagnosis of raised ICP, its effectiveness in improving outcomes has been questioned by the recently published BEST TRIP (Benchmark Evidence from South American Trials: Treatment of Intracranial Pressure) trial. ${ }^{5}$ Further, resource limitations may preclude the utilization of ICP monitoring, especially in low- and middle-income countries.

In this context, we conducted this study to develop a validated set of indications to start treatment for intracranial hypertension in environments where resource limitations preclude invasive ICP monitoring. The same indications can also be used to select patients for invasive ICP monitoring in high-resource settings.

\section{Methods \\ Study Design}

The set of potential predictors that we tested arose via a Delphi-based consensus development process. ${ }^{10}$ The consensus group involved 43 neurosurgeons and intensivists who have extensive experience managing patients with severe TBI based on clinical examination and CT findings alone without ICP monitoring. They proposed a set of predictors, based on CT and clinical findings at baseline after resuscitation, to identify those patients for whom they would recommend initiating treatment for suspected intracranial hypertension under conditions without ICP monitoring. This process was part of an NIH clinical research study funded through the National Institute of Neurological Disorders and Stroke (NINDS) and the Fogarty International Center. The study (Managing Severe Traumatic Brain Injury [TBI] Without Intracranial Pressure Monitoring [ICP] Monitoring Guidelines) is registered with the clinicaltrials.gov database, and its registration number is NCT02059941. The details of the Delphi consensus process were published elsewhere. ${ }^{10}$ To validate the clinical decision rule, we used individual patient data from 2 randomized controlled trials conducted in different settings (see Data Source, below). This study was determined exempt from review by the institutional review board at the University of Washington, Seattle, Washington.

\section{Data Source}

In the primary analysis, we used data from the BEST TRIP randomized controlled trial $(n=324$; clinicaltrials. gov identifier: NCT01068522). This trial prospectively compared the treatment of severe TBI patients using a protocol based on invasive ICP monitoring $(n=157)$ versus an alternative protocol based on imaging and clinical examination without monitoring $(n=167)$ in 10 hospitals from low- and middle-income countries in Latin America. In a secondary analysis, we also validated the clinical decision rule in a separate dataset from the Citicoline Brain Injury Treatment Trial (COBRIT; clinicaltrials. gov identifier: NCT00545662) to ensure generalizability to populations of high-income countries (HICs). ${ }^{20}$ The COBRIT trial was a multicenter, double-blinded, randomized trial involving 1213 patients at 8 Level I trauma centers in the US to investigate effects of citicoline versus placebo in patients with complicated mild, moderate, or severe TBI. ${ }^{20}$

\section{Assembly of Validation Cohort}

We identified patients enrolled in the trials who were 13 years of age or older with a total Glasgow Coma Scale (GCS) score $\leq 8$ on admission or within 48 hours after injury. Only patients who underwent invasive ICP monitoring were included in the validation cohort. Patients with foreign objects in the brain parenchyma, a GCS score of 3 and bilateral fixed and dilated pupils, or unsurvivable injuries were excluded from the trials.

\section{Outcome Measures}

The outcome of interest is intracranial hypertension. In the primary analysis, we defined intracranial hypertension as an hourly ICP reading greater than $22 \mathrm{~mm} \mathrm{Hg}$ in any hour during the ICP monitoring period. In secondary analyses, we defined intracranial hypertension as an ICP greater than $25 \mathrm{~mm} \mathrm{Hg}$ and greater than $30 \mathrm{~mm} \mathrm{Hg}$ in any hour during the monitoring period.

\section{Statistical Analysis}

Descriptive statistics were calculated for all patients included in our validation cohort. We compared the baseline characteristics of patients who had intracranial hypertension with those of patients who did not. We used the chi-square test with continuity correction or Fisher test, as appropriate, to compare proportions, and Wilcoxon ranksum test to compare distributions.

To assess the discrimination and calibration of the proposed predictors, we created a binary logistic regression model with the dependent variable of intracranial hypertension modeled relative to the predictors. To account for 
TABLE 1. Clinical decision rule for prediction of intracranial hypertension in severe TBI

\begin{tabular}{l} 
High ICP should be suspected \& ICP monitoring \&/or ICP-lowering \\
treatment initiated in the presence of 1 major or 2 minor criteria. \\
\hline Major criteria \\
\hline Compressed cisterns (Marshall DI III) \\
\hline Midline shift $>5 \mathrm{~mm}$ (Marshall DI IV) \\
\hline Nonevacuated mass lesion $\left(>25 \mathrm{~cm}^{3}\right)$ \\
\hline Minor criteria \\
\hline GCS motor score $\leq 4$ \\
\hline Pupillary asymmetry \\
\hline Abnormal pupillary reactivity \\
\hline Marshall DI II (i.e., basal cisterns are present w/ midline shift of \\
$0-5$ mm \&/or high- or mixed-density lesion of $\geq 25 \mathrm{~cm}^{3}$ ) \\
\hline
\end{tabular}

$\mathrm{DI}=$ diffuse injury; GCS = Glasgow Coma Scale; ICP = intracranial pressure;

$\mathrm{TBI}=$ traumatic brain injury.

missing values for pupillary asymmetry $(3.3 \%)$ and reactivity (24.6\%), a multiple imputations procedure with 10 iterations was performed using the Markov chain Monte Carlo method..$^{16}$ This imputation method is considered less susceptible to bias than performing a complete case analysis by dropping cases with incomplete variables. ${ }^{12}$ The model was tested through the construction of receiver operating characteristic (ROC) curves, for each of the imputation iterations. The area under the curve (AUC) is a summary measure of the discriminative ability of the model, with higher values indicative of better predictive discrimination. AUC values from each of the imputation iterations were averaged to create a single value quantifying model discrimination. Calibration was tested using the Hosmer-Lemeshow goodness-of-fit test.

To report on the performance of the clinical decision rule, we calculated sensitivity, specificity, positive and negative predictive values, and positive and negative likelihood ratios with $95 \%$ confidence intervals (CIs) for the proposed rule (based on clinical and imaging findings at baseline after resuscitation) to predict intracranial hypertension. In secondary analyses, we assessed the performance of the clinical decision rule at definitions of intracranial hypertension using higher ICP thresholds, defining intracranial hypertension as ICP $>25 \mathrm{~mm} \mathrm{Hg}$ and ICP > $30 \mathrm{~mm} \mathrm{Hg}$. Finally, we examined the performance of the rule, using postoperative clinical and imaging variables, in predicting postoperative intracranial hypertension among patients who underwent craniotomy to evacuate a mass lesion or craniectomy. To define postoperative clinical variables, we used the first reported postoperative examination findings as long as they were obtained at least 6 hours after the operation to minimize the potential confounding effect of general anesthetic drugs. A mass lesion was defined as an epidural hematoma, a subdural hematoma, a contusion, or an intracerebral hematoma. Only postoperative variables (i.e., postoperative clinical findings and CT scans) were used to predict intracranial hypertension in this subgroup of patients.

In sensitivity analyses, we assessed the performance of the clinical decision rule derived from admission variables after excluding patients who underwent cranial surgery from the validation cohort. We also assessed the performance of the rule in predicting postoperative intracranial hypertension at varying ICP thresholds to define intracranial hypertension. Finally, we examined performance of the rule after excluding patients who underwent decompressive craniectomy from the postoperative subgroup.

To ensure that the clinical decision rule can be generalized to populations of HICs, we validated the rule using a dataset from the North American COBRIT trial in a secondary analysis using the same methods to define predictors and outcomes.

All statistical analyses were performed using SAS software version 9.3 (SAS Institute, Inc.). All tests were 2 -sided, and $p$ values less than 0.05 were considered to be statistically significant.

\section{Results}

\section{Predictors of Intracranial Hypertension}

The consensus working group proposed a set of predictors on the basis of which to suspect intracranial hypertension. They divided the predictors into major and minor criteria. The major criteria are: 1) compressed cisterns (CT classification of Marshall diffuse injury [DI] III), ${ }^{13}$ 2) midline shift greater than $5 \mathrm{~mm}$ (Marshall DI IV), ${ }^{13}$ and 3) nonevacuated mass lesion $\left(>25 \mathrm{~cm}^{3}\right)$. The minor criteria are: 1) GCS motor score of 4 or less, 2) pupillary asymmetry (difference in diameter between the 2 pupils of more than $1 \mathrm{~mm}$ ), 3) abnormal pupillary reactivity (lack of reactivity to light in one or both pupils), and 4) CT classification of Marshall DI II (i.e., basal cisterns are present with midline shift of $0-5 \mathrm{~mm}$ and/or high- or mixed-density lesion of $25 \mathrm{~cm}^{3}$ or less). ${ }^{13}$

All predictors are to be obtained at baseline following the resuscitation of severe TBI patients.

\section{Clinical Decision Rule}

High ICP would be suspected and ICP monitoring and/ or ICP-lowering treatment should be initiated in the presence of 1 major or 2 or more minor criteria (Table 1). The final rule was approved by $97 \%$ of the participants in the consensus development process.

\section{Characteristics of Latin American Validation Cohort}

The final cohort with ICP data consisted of 150 patients with a median age of 28 years and median total and motor GCS scores of 7 and 5, respectively (Table 2). In contrast to patients without intracranial hypertension, patients who suffered high ICP were younger and more likely to have abnormal pupillary response to light or diffuse injury IIIV as measured by the Marshall CT classification system (Table 2 and Supplementary Appendix Table S1).

\section{Performance of the Predictors}

For the logistic model containing the proposed predictors, the AUC value averaged across the 10 imputation iterations was 0.86 ( 0.81 in the complete case analysis). The $\mathrm{p}$ value for the Hosmer-Lemeshow goodness-of-fit test was 0.49 ( 0.54 in the complete case analysis). 
TABLE 2. Baseline characteristics of the Latin American validation cohort

\begin{tabular}{|c|c|c|c|c|}
\hline Characteristic & $\begin{array}{l}\text { Overall } \\
\text { Cohort (n } \\
=150)\end{array}$ & $\begin{array}{c}\text { High ICP } \\
\text { Group* } \\
(n=65)\end{array}$ & $\begin{array}{l}\text { Normal } \\
\text { ICP Group } \\
(\mathrm{n}=85)\end{array}$ & $\begin{array}{c}p \\
\text { Value }\end{array}$ \\
\hline Age in yrs & & & & 0.009 \\
\hline Median & 28 & 25 & 31 & \\
\hline IQR & $21-44$ & $20-34$ & $22-48$ & \\
\hline Female sex & $12(8)$ & $4(6.2)$ & $8(9.4)$ & 0.55 \\
\hline Initial GCS score & & & & 0.69 \\
\hline Median & 7 & 7 & 7 & \\
\hline IQR & $5-9$ & $6-9$ & $5-9$ & \\
\hline Initial motor GCS score & & & & 0.91 \\
\hline Median & 5 & 4 & 5 & \\
\hline IQR & $3-5$ & $3-5$ & $3-5$ & \\
\hline $\begin{array}{l}\text { Pupillary symmetry } \dagger \\
\text { on admission }\end{array}$ & & & & 0.37 \\
\hline Normal & $109(72.6)$ & $51(78.5)$ & $58(68.2)$ & \\
\hline Asymmetrical & $36(24)$ & $12(18.5)$ & $24(28.2)$ & \\
\hline Unknown & $5(3.3)$ & $2(3.1)$ & $3(3.5)$ & \\
\hline $\begin{array}{l}\text { Pupillary reactivity } \ddagger \text { on } \\
\text { admission }\end{array}$ & & & & 0.05 \\
\hline Normal & $71(47.3)$ & $24(36.9)$ & $47(55.2)$ & \\
\hline Abnormal & $42(28)$ & $24(36.9)$ & $18(21.2)$ & \\
\hline Unknown & $37(24.7)$ & $17(26.2)$ & $20(23.5)$ & \\
\hline $\begin{array}{l}\text { Marshall classification } \\
\text { on initial CT }\end{array}$ & & & & $<0.0001$ \\
\hline DIII & $21(14)$ & $13(20)$ & $8(9.4)$ & \\
\hline DI III & $70(46.7)$ & $40(60.5)$ & $30(35.3)$ & \\
\hline DI IV & $9(6)$ & $6(9.2)$ & $3(3.5)$ & \\
\hline $\begin{array}{l}\text { Evacuated mass } \\
\text { lesion }\end{array}$ & $48(32)$ & $4(6.2)$ & $44(51.8)$ & \\
\hline $\begin{array}{l}\text { Nonevacuated } \\
\text { mass lesion }\end{array}$ & $2(1.3)$ & $2(3.1)$ & $0(0)$ & \\
\hline
\end{tabular}

$\mathrm{IQR}=$ interquartile range.

Data are number of patients (\%) unless otherwise indicated.

* Defined as ICP $>22 \mathrm{~mm} \mathrm{Hg}$ at any point during monitoring prior to any neurosurgical intervention.

$\dagger$ Pupillary asymmetry was defined as a difference in diameter between the 2 pupils of more than $1 \mathrm{~mm}$.

$\ddagger$ Abnormal pupillary response was defined as lack of reactivity to light in one or both pupils.

\section{Validation of the Clinical Decision Rule in the Latin American Cohort}

When high ICP was defined as $>22 \mathrm{~mm} \mathrm{Hg}$, the decision rule performed with a sensitivity of $93.9 \%(95 \% \mathrm{CI}$ $85.0 \%-98.3 \%$ ), a specificity of $42.3 \%$ (95\% CI $31.7 \%-$ $53.6 \%)$, a positive predictive value of $55.5 \%(95 \% \mathrm{CI}$ $50.7 \%-60.2 \%$, and a negative predictive value of $90 \%$ (95\% CI 77.1\%-96.0\%) (Table 3).

\section{Secondary Analyses}

The sensitivity of the clinical decision rule improved with higher ICP cutoffs (Table 3 ) up to a sensitivity and negative predictive value of $100 \%$ when intracranial hypertension was defined as ICP $>30 \mathrm{~mm} \mathrm{Hg}$. Among patients who underwent craniotomy for evacuation of a mass lesion or craniectomy $(n=69$, Table S1, Supplementary Appendix), the clinical decision rule for predicting postoperative intracranial hypertension based on postoperative clinical and imaging findings had a sensitivity of $80 \%$ and a specificity of $9.3 \%$ (Table 4 ).

\section{Sensitivity Analyses}

Similar results were found after excluding patients who underwent cranial surgery (Table S5, Supplementary Appendix) from the validation cohort. For predicting postoperative intracranial hypertension, the performance of the clinical decision rule did not change significantly when the ICP threshold was changed to 25 or $30 \mathrm{~mm} \mathrm{Hg}$ (Table S6, Supplementary Appendix) or after excluding decompressive craniectomy patients (Table S7, Supplementary Appendix).

\section{Validation of the Clinical Decision Rule in the North American Cohort}

Similar results were found in the North American cohort. When high ICP was defined as $>22 \mathrm{~mm} \mathrm{Hg}$, the decision rule performed with a sensitivity of $93.6 \%(95 \%$ CI $85.7 \%-97.9 \%$ ) and a specificity of $34.0 \%$ (95\% CI $21.5 \%-48.3 \%$ ) (Table S9, Supplementary Appendix). The sensitivity of the rule increased to $95.7 \%$ at the higher ICP threshold of $30 \mathrm{~mm} \mathrm{Hg}$ (i.e., when intracranial hypertension was defined as ICP > $30 \mathrm{~mm} \mathrm{Hg}$ ).

\section{Discussion}

Using the Delphi consensus method, a large multidisciplinary group of experienced neurosurgeons and intensivists from resource-limited countries identified a set of predictors derived from basic clinical and imaging findings that defined those patients whom they would treat for suspected intracranial hypertension in the absence of ICP monitoring..$^{10}$ Based on these predictors, we proposed and validated in 2 different populations a clinical decision rule that is clinically sensible, easy to apply, and highly sensitive for the prediction of intracranial hypertension events on arrival to the hospital, especially at high thresholds for defining raised ICP. However, the performance of the decision rule using postoperative data in predicting postoperative intracranial hypertension was limited by modest sensitivity and poor specificity.

Management of intracranial hypertension is considered the cornerstone of modern care for severe TBI. Accurate and continuous ICP monitoring via an invasive tool can lead to the prompt recognition of spiking pressure around the injured parts of the brain..$^{14}$ Timely recognition would facilitate expedient intervention to control the rising pressure. ${ }^{17,19}$ It is unclear, however, how to predict a rise in ICP or when to start monitoring and treatment. This challenge is further complicated in environments with limited resources that preclude the utilization of ICP monitors.

The recently updated Brain Trauma Foundation guidelines did not carry forward the traditional indications for ICP monitoring, because they were derived from a de- 
TABLE 3. Performance of the clinical decision rule in the Latin American cohort

\begin{tabular}{|c|c|}
\hline & Result \\
\hline \multicolumn{2}{|l|}{ When high ICP is defined as $>22 \mathrm{~mm} \mathrm{Hg}$} \\
\hline Rule positive & Pts w/ high ICP: $n=61 ;$ pts $w /$ normal ICP: $n=49$ \\
\hline Rule negative & Pts w/ high ICP: $n=4 ;$ pts w/ normal ICP: $n=36$ \\
\hline Sensitivity & $93.9 \%(95 \% \mathrm{Cl} 85.0-98.3 \%)$ \\
\hline Specificity & $42.4 \%(95 \% \mathrm{Cl} 31.7-53.6 \%)$ \\
\hline Positive predictive value & $55.5 \%(95 \% \mathrm{Cl} 50.7-60.2 \%)$ \\
\hline Negative predictive value & $90.0 \%(95 \% \mathrm{Cl} 77.1-96.0 \%)$ \\
\hline Positive likelihood ratio & $1.6(95 \% \mathrm{Cl} 1.3-2.0)$ \\
\hline Negative likelihood ratio & $0.2(95 \% \mathrm{Cl} 0.1-0.4)$ \\
\hline AUC for logistic regression model that contains all the predictors & 0.861 \\
\hline Hosmer-Lemeshow goodness-of-fit test & $p=0.49$ \\
\hline \multicolumn{2}{|l|}{ When high ICP is defined as $>25 \mathrm{~mm} \mathrm{Hg}$} \\
\hline Rule positive & Pts w/ high ICP: $n=50 ;$ pts $w /$ normal ICP: $n=60$ \\
\hline Rule negative & Pts w/ high ICP: $n=1 ;$ pts $w /$ normal ICP: $n=39$ \\
\hline Sensitivity & $98.0 \%(95 \% \mathrm{Cl} 89.6-100.0 \%)$ \\
\hline Specificity & $39.4 \%(95 \% \mathrm{Cl} 29.7-49.7 \%)$ \\
\hline Positive predictive value & $45.5 \%(95 \% \mathrm{Cl} 41.4-49.5 \%)$ \\
\hline Negative predictive value & $97.5 \%(95 \% \mathrm{Cl} 84.7-99.6 \%)$ \\
\hline Positive likelihood ratio & $1.6(95 \% \mathrm{Cl} 1.4-1.9)$ \\
\hline Negative likelihood ratio & $0.1(95 \% \mathrm{Cl} 0.0-0.4)$ \\
\hline AUC for logistic regression model that contains all the predictors & 0.83 \\
\hline Hosmer-Lemeshow goodness-of-fit test & $p=0.36$ \\
\hline \multicolumn{2}{|l|}{ When high ICP is defined as $>30 \mathrm{~mm} \mathrm{Hg}$} \\
\hline Rule positive & Pts w/ high ICP: $n=40 ;$ pts $w /$ normal ICP: $n=70$ \\
\hline Rule negative & Pts w/ high ICP: $n=0 ;$ pts w/ normal ICP: $n=40$ \\
\hline Sensitivity & $100.0 \%(95 \% \mathrm{Cl} 91.2-100.0 \%)$ \\
\hline Specificity & $36.4 \%(95 \% \mathrm{Cl} 27.4-46.1 \%)$ \\
\hline Positive predictive value & $36.4 \%(95 \% \mathrm{Cl} 33.2-39.7 \%)$ \\
\hline Negative predictive value & $100.0 \%(95 \% \mathrm{Cl} 89.1-100.0 \%)$ \\
\hline Positive likelihood ratio & $1.6(95 \% \mathrm{Cl} 1.4-1.8)$ \\
\hline Negative likelihood ratio & $0.0(95 \% \mathrm{Cl} 0.0-0.0)$ \\
\hline AUC for logistic regression model that contains all the predictors & 0.82 \\
\hline Hosmer-Lemeshow goodness-of-fit test & $p=0.72$ \\
\hline
\end{tabular}

Pts = patients.

TABLE 4. Performance of the clinical decision rule in the postoperative subgroup of Latin American cohort

\begin{tabular}{ll}
\hline & \multicolumn{1}{c}{ Result } \\
\hline Rule positive & Pts w/ high ICP:* $n=12 ;$ pts w/ normal ICP: $n=49$ \\
\hline Rule negative & Pts w/ high ICP:* $n=3 ;$ pts w/ normal ICP: $n=5$ \\
\hline Sensitivity & $80 \%(95 \% \mathrm{Cl} 51.9-95.7 \%)$ \\
\hline Specificity & $9.3 \%(95 \% \mathrm{Cl} 3.1-20.3 \%)$ \\
\hline Positive predictive value & $19.7 \%(95 \% \mathrm{Cl} 15.8-24.2 \%)$ \\
\hline Negative predictive value & $62.5 \%(95 \% \mathrm{Cl} 31.0-86.1 \%)$ \\
\hline Positive likelihood ratio & $0.9(95 \% \mathrm{Cl} \mathrm{0.7-1.2)}$ \\
\hline Negative likelihood ratio & $2.2(95 \% \mathrm{Cl} \mathrm{0.6-8.0)}$ \\
\hline AUC for logistic regression model that contains all the predictors & 0.65 \\
\hline Hosmer-Lemeshow goodness-of-fit test & $\mathrm{p}=0.96$ \\
\hline
\end{tabular}

${ }^{*}$ High ICP was defined as $>22 \mathrm{~mm} \mathrm{Hg}$. 
scriptive study of patient characteristics associated with risk of high ICP and were never validated., ${ }^{1,3,15}$ In addition, the BEST TRIP trial found no outcome differences between a protocol based on ICP monitoring and an alternative protocol based on imaging and clinical examination without monitoring as applied to the collective group of all patients with severe TBI. Therefore, it can be argued that the imaging and clinical examination protocol is an acceptably effective option, especially in low- and middleincome countries. However, the absence of a published protocol for managing severe cases of TBI without ICP monitoring necessitates elaborating and validating one. Our study addressed the first step in building such a protocol by providing a tool to predict and initiate treatment for intracranial hypertension.

This clinical decision rule was developed and approved by consensus among a large group of clinicians who have extensive experience in managing cases of severe TBI without monitoring. ${ }^{10}$ The rule is based on sensible clinical and CT scan variables that can be easily measured by the intended users upon patients' arrival to the hospital. It was designed to achieve a high level of sensitivity and performed at this sensitivity level during the validation part of this study. As with most clinical decision rules that relate to a potentially serious outcome, we believe that the high sensitivity is the most important characteristic of the rule to be sought after, because we are trying to ensure that patients do not have increased morbidity or mortality as the result of a missed intracranial hypertension event.

We do not believe that the relatively modest specificity and positive predictive value of the decision rule would lead to adverse events in cases in which the rule is falsely positive. The results of the BEST TRIP trial have suggested that ICP-lowering therapy is relatively benign. Although the trial group that was treated without ICP monitoring received more ICP-lowering interventions for longer periods of time, the patients did not suffer higher rates of adverse events, and the long-term outcomes in that group were similar to those in the ICP-monitored group, which received fewer ICP-lowering interventions. ${ }^{5}$

This clinical decision rule is not intended to limit the individualization of management appropriate for each patient or to impose a particular treatment structure on clinicians. The subsequent ideal step after a patient is identified by this rule might be to monitor ICP invasively in environments in which such monitoring is feasible. In resource-poor environments, one possible subsequent step might be initiating a low-risk treatment as a safe and more practical approach given the lack of invasive ICP monitoring tools. Another potential option is closer serial clinical assessment and more frequent CT scanning. The ultimate choice of subsequent course of action depends on individual patients' characteristics and clinicians' judgment of risk-to-benefit ratio.

Our study has several limitations. The validation cohorts were derived retrospectively from 2 randomized controlled trials conducted in Latin America and North America, which may not be representative of the entire spectrum of the severe TBI population. Consecutive patients, however, were prospectively enrolled in these pragmatic multicenter trials, and high-quality data were prospectively collected after extensive training of site personnel and un- der close monitoring in both trials. ${ }^{2,5}$ Another limitation is that clinical examination data, including pupillary findings and CT scan data, were reported by the treating clinicians. It is possible that individual clinicians interpret pupillary size and reactivity and CT scans differently. However, a previous study has shown good interobserver reproducibility between neurosurgeons and neuroradiologists in categorizing CT scans according to the Marshall classification system. ${ }^{6}$ Further, this decision rule is meant to be a feasible tool that can be used by clinicians caring for TBI patients acutely at the bedside in a timely fashion. A third limitation is the modest sensitivity of the rule in predicting postoperative intracranial hypertension. It is possible that the relatively small sample size of postoperative patients in the trial is not representative of the general postoperative TBI population. Another potential explanation is the difficulty in predicting intracranial hypertension in this patient subgroup without accounting for the details of the surgical procedure and/or without more detailed CT scan assessment. Further studies are needed to address the prediction of postoperative intracranial hypertension in this subgroup of TBI patients who had craniotomy or craniectomy for mass lesion evacuation. The evolution of serial clinical examination and imaging data is another potential variable that might help better predict intracranial hypertension and should be examined in future research.

The sensitivity of the rule was high at the traditional ICP cutoff used to define intracranial hypertension. However, it reached $100 \%$ only with a threshold of $30 \mathrm{~mm} \mathrm{Hg}$. We believe this is an opportunity to further question the value of a universal fixed threshold for defining intracranial hypertension rather than an actual limitation of the rule. First, the stepwise progressive increase in sensitivity with higher thresholds in 2 different populations lends further credence to the underlying rationale, face validity, and clinical sensibility of the rule. Second, the BEST TRIP trial showed no difference in long-term efficacy between a protocol based on ICP monitoring and a fixed universal value to define raised ICP and a protocol based on imaging and clinical variables alone (using clinical variables similar to the ones included in this decision rule).

The BEST TRIP trial did not address the efficacy of ICP monitor-based treatment of patients with actual, demonstrated intracranial hypertension (using any threshold). ${ }^{4}$ Accordingly, we suggest that the utility of this decision rule in situations where ICP monitoring is available would be to assist in determining whom to monitor rather than whom to treat for suspected intracranial hypertension.

Future studies are needed to validate this rule in other populations. The demographic and injury characteristics of different populations might impact the performance of the rule. In addition, this rule does not address relevant practical issues, such as when to taper or escalate ICPlowering therapy. Future research should answer these important questions.

\section{Conclusions}

A simple clinical decision rule based on a combination of clinical and imaging findings was validated in 2 different populations and found to be highly sensitive in distin- 
guishing severe TBI patients who would suffer intracranial hypertension. It could be used to identify patients who require ICP monitoring in high-resource settings or start ICP-lowering treatment in environments where resource limitations preclude invasive monitoring

\section{Acknowledgments}

The consensus conference was supported by funds from the National Institutes of Health/the National Institute of Neurological Disorders and Stroke (NIH/NINDS) (1R01NS080648) and the Fogarty International Center; ClinicalTrials.gov (NCT02059941). The BEST TRIP trial was funded by the NIH/NINDS (1R01NS058302) and others; ClinicalTrials.gov (NCT01068522). The COBRIT trial was supported by Eunice Kennedy Shriver National Institute of Child Health and Human Development and others; ClinicalTrials.gov (NCT00545662). The content is solely the responsibility of the authors and does not necessarily represent the official views of the National Institutes of Health.

We would like to thank those members of the consensus conference investigator group who are not included in the authorship of the current paper: Perla Blanca Pahnke, MD, Hospital Municipal de Urgencias, Cordoba, Argentina; Daniel Godoy, MD, Sanatorio Pasteur, Catamarca, Argentina; Gustavo Pinero, MD, Leonidas, Lucero Hospital, Bahia Blanca, Argentina; Freddy Sandi Lora, MD, Hospital Obrero No. 1 de La Paz, La Paz, Bolivia; Sergio Aguilera, MD, Hospital Regional, Inquique, Chile; Andres Rubiano, MD, Meditech Foundation, El Bosque University, Neiva, Colombia; Caridad Soler Morejon, MD, Ameijeiras Hospital, La Habana, Cuba; Manuel Jibaja, MD, Hospital Eugenio Espejo, Quito, Ecuador; Hubiel Lopez, MD, Hospital General de la Plaza de la Salud, Santo Domingo, Dominican Republic; and Ricardo Romero, MD, Fundación Clinica Campbell, Barranquilla, Colombia.

We wish to acknowledge Joanie Howard of Electronic Media Services Inc. for providing and running the automatic response system used in voting and Ezequiel L. Russo, Cristina Gonzalez, and Silvia Calens for serving as multilingual facilitators during the meeting. We would also like to acknowledge the Integra Foundation, as funds from the Integra Endowed Professorship in Neurotrauma (held by R. M. Chesnut at the University of Washington) were used in support of the consensus conference meeting. Finally, we would like to thank those of the BEST TRIP trial investigators and study staff who are not among the authors of the current paper-C. Rondina and N. Carney (investigators) and J. Machamer, J. Celix, M. Cherner, and T. Hendrix (study staff) - as well as those of the COBRIT trial investigators who are not among the authors of the current paper-R. D. Zafonte, DO; E. Bagiella, PhD; B. M. Ansel, PhD; T. A. Novack, PhD; W. T. Friedewald, MD; D. C. Hesdorffer, PhD; S. D. Timmons, MD; J. Jallo, MD, $\mathrm{PhD} ;$ H. Eisenberg, MD; T. Hart, PhD; J. H. Ricker, PhD; R. DiazArrastia, MD, PhD; R. E. Merchant, PhD; and S. Melton, MD.

\section{References}

1. Bratton SL, Chestnut RM, Ghajar J, McConnell Hammond FF, Harris OA, Hartl R, et al: Guidelines for the management of severe traumatic brain injury. VI. Indications for intracranial pressure monitoring. J Neurotrauma 24 (Suppl 1):S37S44, 2007

2. Carney N, Lujan S, Dikmen S, Temkin N, Petroni G, Pridgeon J, et al: Intracranial pressure monitoring in severe traumatic brain injury in Latin America: process and methods for a multi-center randomized controlled trial. J Neurotrauma 29:2022-2029, 2012

3. Carney N, Totten AM, O'Reilly C, Ullman JS, Hawryluk GW, Bell MJ, et al: Guidelines for the Management of Severe Traumatic Brain Injury, Fourth Edition. Neurosurgery 80:6-15, 2017
4. Chesnut RM, Bleck TP, Citerio G, Classen J, Cooper DJ, Coplin WM, et al: A consensus-based interpretation of the Benchmark Evidence from South American Trials: Treatment of Intracranial Pressure trial. J Neurotrauma 32:1722-1724, 2015

5. Chesnut RM, Temkin N, Carney N, Dikmen S, Rondina C, Videtta W, et al: A trial of intracranial-pressure monitoring in traumatic brain injury. N Engl J Med 367:2471-2481, 2012

6. Chun KA, Manley GT, Stiver SI, Aiken AH, Phan N, Wang $\mathrm{V}$, et al: Interobserver variability in the assessment of CT imaging features of traumatic brain injury. J Neurotrauma 27:325-330, 2010

7. Eisenberg HM, Frankowski RF, Contant CF, Marshall LF, Walker MD: High-dose barbiturate control of elevated intracranial pressure in patients with severe head injury. J Neurosurg 69:15-23, 1988

8. Feigin VL, Theadom A, Barker-Collo S, Starkey NJ, McPherson K, Kahan M, et al: Incidence of traumatic brain injury in New Zealand: a population-based study. Lancet Neurol 12:53-64, 2013

9. Finkelstein EACP, Miller TR: The Incidence and Economic Burden of Injuries in the United States. New York: Oxford University Press, 2006

10. Hendrickson P, Pridgeon J, Temkin NR, Videtta W, Petroni G, Lujan S, et al: Development of a severe traumatic brain injury consensus-based treatment protocol conference in Latin America. World Neurosurg 110:e952-e957, 2018

11. Hyder AA, Wunderlich CA, Puvanachandra P, Gururaj G, Kobusingye OC: The impact of traumatic brain injuries: a global perspective. NeuroRehabilitation 22:341-353, 2007

12. Little, RJA: Regression with missing X's: a review. J Am Stat Assoc 87:1227-1237, 1992

13. Marshall LF, Marshall SB, Klauber MR, Clark MvB, Eisenberg HM, Jane JA, et al: A new classification of head injury based on computerized tomography. J Neurosurg 75:S14S20, 1991

14. Miller JD, Becker DP, Ward JD, Sullivan HG, Adams WE, Rosner MJ: Significance of intracranial hypertension in severe head injury. J Neurosurg 47:503-516, 1977

15. Narayan RK, Kishore PR, Becker DP, Ward JD, Enas GG, Greenberg RP, et al: Intracranial pressure: to monitor or not to monitor? A review of our experience with severe head injury. J Neurosurg 56:650-659, 1982

16. Oyetunji TA, Crompton JG, Ehanire ID, Stevens KA, Efron DT, Haut ER, et al: Multiple imputation in trauma disparity research. J Surg Res 165:e37-e41, 2011

17. Park E, Bell JD, Baker AJ: Traumatic brain injury: can the consequences be stopped? CMAJ 178:1163-1170, 2008

18. Rubiano AM, Carney N, Chesnut R, Puyana JC: Global neurotrauma research challenges and opportunities. Nature 527:S193-S197, 2015

19. Sahuquillo J, Arikan F: Decompressive craniectomy for the treatment of refractory high intracranial pressure in traumatic brain injury. Cochrane Database Syst Rev (1):CD003983, 2006

20. Zafonte RD, Bagiella E, Ansel BM, Novack TA, Friedewald WT, Hesdorffer DC, et al: Effect of citicoline on functional and cognitive status among patients with traumatic brain injury: Citicoline Brain Injury Treatment Trial (COBRIT). JAMA 308:1993-2000, 2012

21. Zygun DA, Laupland KB, Hader WJ, Kortbeek JB, Findlay $\mathrm{C}$, Doig CJ, et al: Severe traumatic brain injury in a large Canadian health region. Can J Neurol Sci 32:87-92, 2005

\section{Disclosures}

The authors report no conflict of interest concerning the materi- 
als or methods used in this study or the findings specified in this paper.

\section{Author Contributions}

Conception and design: Alali, Temkin, Dikmen, Hendrickson, Videtta, Lujan, Petroni, Guadagnoli, Urbina, Chesnut. Acquisition of data: Alali, Temkin, Barber, Chaddock, Dikmen, Hendrickson, Videtta, Lujan, Petroni, Guadagnoli, Chesnut. Analysis and interpretation of data: Alali, Temkin, Barber, Chesnut. Drafting the article: Alali, Pridgeon, Chaddock. Critically revising the article: Alali, Temkin, Pridgeon, Chaddock, Chesnut. Reviewed submitted version of manuscript: Alali, Temkin, Pridgeon, Chaddock, Dikmen, Hendrickson, Videtta, Lujan, Petroni, Guadagnoli, Chesnut. Approved the final version of the manuscript on behalf of all authors: Alali. Statistical analysis: Alali, Temkin, Barber. Administrative/technical/material support: Temkin, Chaddock, Chesnut. Study supervision: Temkin, Chesnut.

\section{Supplemental Information}

Online-Only Content

Supplemental material is available with the online version of the article.

Supplementary Appendix. https://thejns.org/doi/suppl/10.3171/ 2018.4.JNS173166.

\section{Correspondence}

Aziz S. Alali: University of Washington, Seattle, WA. aalali@ uw.edu. 